\title{
Computer Information Processing Technology in the Age of Big Data
}

\author{
Emilin Mathew ${ }^{1}$ \\ ${ }^{1}$ Plano West Senior High School, Plano, Texas
}

\begin{abstract}
Under the background of big data times, information technology means emerge endlessly, and the value of technology application is improved gradually. At present, each industry gradually applies the information processing technology related to computer system to carry out the work of information resource processing. With the support of computer equipment, the processing of information resources is more efficient and accurate. In the process of computer application, the computer information system should be optimized and perfected by many technical means, and the application standard of each technique should also be defined, so that the accuracy and reliability of information processing can be further enhanced. Based on this, this paper will discuss the computer information processing technology in the big data age.
\end{abstract}

Keywords: Big data age; Computers; Information processing technology

\section{Introduction}

In the process of vigorous development of science and technology, computer information technology presents the trend of rapid development, meanwhile, the information system in the era of big data is developing towards more intellectualization and modernization. Computer information processing technology should be based on the current needs of use, strengthen the security of the information process, improve the efficiency of processing information, so that the quality of data can be improved, further optimize the service quality, and thus lay the foundation for the sustainable development of various fields of industry.

\section{Application Features of Computer Information Processing Technology in the Age of Large Data}

With the advent of the big data era, the information processing work has been significantly affected. Facing various data application environments, data processing systems display different advantages, which can provide more comprehensive services for data management. The application characteristics of computer information processing technology are as follows:

\subsection{More flexible and comprehensive coverage}

The processing of data resources through computer equipment not only makes the data more comprehensive, but also requires flexibility in order to meet the needs of many different types of clients. The current database is also characterized by openness, access to the acquired data, a comprehensive analysis from multiple perspectives, and a greater understanding of the user and hence a significant improvement in the original efficiency of processing.

\subsection{Modular operating model}

The data processing system can be run in a modular way, can distinguish data effectively, and can separate and integrate data with processing requirements. The data processing system is composed of many different modules, and the functions of these modules can be displayed during the processing of data, which can effectively handle and manage the data. In the data processing system, each mode runs independently and does not affect each other. When performing special data processing tasks, the modules can also be run in a coordinated manner, thus providing a guarantee for the smooth implementation of other information applications.

\subsection{An open data processing environment}

Under the background of big data age, the utilization value of data resource is rich and the data amount is huge day by day. At the same time, the data processing environment also presents the characteristics of open development. The collection and utilization of data and information can be carried out at any time and place with the support of information equipment such as computers. When using computers to process data resources, it needs to cooperate 
with hardware and software facilities. At present, all kinds of software and hardware are compatible with each other, even if the software is installed in other hardware platforms, and almost all third-party programs have external interfaces built into them. Therefore, data information processing is more convenient. In addition, the software market is relatively flexible, enriching its capabilities on the basis of hardware devices, allowing it to further expand its range of functions and thus achieve efficient processing of data in a more open environment.

\section{Analysis of computer information processing techniques in the age of big data}

Using computer platform to process information data needs to use and manage information completely differently from different information processing tasks.

\subsection{Information collection and processing technologies}

In the process of data information processing, the computer is the most critical technology platform. The computer has strong function and can use many kinds of information service functions to effectively collect and process the data, at the same time, it can transmit data quickly. After the collection of information data, computer equipment should be used to analyze the data comprehensively, extract valuable data and then apply it pertinently. Data processing is one of the important functions of computer equipment, which can be analyzed based on data content, classified according to its external form, effectively integrate data information with common features, and display data in visual form. The modern data resource transmission technology has the characteristics of strong interactivity and high negotiability, and the data transmission scope is more extensive. In the process of improving computer information processing technology, data transmission will be more secure and efficient, which can lay a good foundation for smooth development of data adjustment.

\subsection{Information security assurance technologies}

Table is as follows: Use words rather than symbols or abData resource enables people to obtain a brand-new way of information transmission. While enjoying the high quality information service brought by the data processing system, they can not ignore the hidden dangers in the process of data utilization and deal with them in time so as to achieve the goal of protecting data resource. In order to solve the problems in data protection, specialized talents should be recruited to take part in the work, the protection system shall be updated at the appropriate time, special protection mechanism should also be set up according to the characteristics of the data, and sufficient development funds must be provided to maintain the security of data transmission process and to ensure the timely detection of risk factors through monitoring devices. In the chain of custody, in addition to protecting against external influences, useless data in computer information systems need to be processed to ensure that the data transmitted is of practical use. Safety and security has become one of the most important tasks in data processing system. Although the big data era has created a more free and stable environment for data transmission, risk factors can not be ignored. Only by comprehensively processing data in many aspects can we ensure that the value of data is not destroyed.

\section{Conclusion}

Information resource is the most valuable resource in the big data era. This paper analyzes the problems in information application, and then analyzes several common information processing technologies. Information systems support many kinds of information processing activities, the total amount of data resources in the big data age is large, and the new data processing requirements formed must be met. Data processing supported by computer platforms still has some defects. Technicians should continue to integrate many platforms to improve the efficiency of processing information resources.

\section{References}

[1] Information and Computer Systems (Theory), 2017, 380 (10): 179-180.

[2] Xiaoli Zhu, Peng Gao. Explore Computer Information Processing Technology in Big Data Age[J]. Science Public (Science Education), 2018 (7): 143. 